\title{
The Role of Screenings Methods and Risk Profile Assessments in Prevention and Health Promotion Programmes: An Ethnographic Analysis
}

\author{
Yvonne J. F. M. Jansen • Antoinette A. de Bont
}

Published online: 10 January 2010

(C) The Author(s) 2009. This article is published with open access at Springerlink.com

\begin{abstract}
In prevention and health promotion interventions, screening methods and risk profile assessments are often used as tools for establishing the interventions' effectiveness, for the selection and determination of the health status of participants. The role these instruments fulfil in the creation of effectiveness and the effects these instruments have themselves remain unexplored. In this paper, we have analysed the role screening methods and risk profile assessments fulfil as part of prevention and health promotion programmes in the selection, enrolment and participation of participants. Our analysis showed, that screening methods and health risk assessments create effects as they objectify health risks and/or the health status of individuals, i.e., they select the individuals 'at risk' and indicate the lifestyle modifications these people are required to make in order to improve their health. Yet, these instruments also reduce the group of participants thereby decreasing the possible effect of interventions, as they provide the legitimisation for people to make choices to whether they enrol or not and what lifestyle changes they incorporate into their lives. In other words, they present a space of interaction, in which agency is distributed across the practice nurses, the participants and the instruments. Decisions were not just made upon the projection of the outcomes of these instruments; decisions that were made by both the patients and practice nurses were the resultant of their opinions on these outcomes that were formed in interaction with the instruments.
\end{abstract}

Y. J. F. M. Jansen · A. A. de Bont

Institute of Health Policy and Management, Department of Healthcare Governance, Erasmus MC, Rotterdam, The Netherlands

\section{Y. J. F. M. Jansen ( $\square)$}

Department Quality of Life, Innovation in Care, TNO Netherlands Organisation for Applied Scientific Research, P.O. Box 2215, 2301 CE Leiden, The Netherlands e-mail: yvonne.jansen@tno.nl 
Keywords Effectiveness - Evaluation of interventions - Legitimisation ·

Pragmatic process of daily care - Prevention and health promotion ·

Public health · Rationalisation · Risk profile assessments - Screening methods

\author{
Abbreviations \\ CVD Cardiovascular diseases \\ GP General practitioner \\ RCT Randomised controlled trial \\ CVA Cardiovascular accident \\ TIA Transient ischemic attack \\ LHV Dutch National Association of General Practitioners
}

\title{
Introduction
}

Scientific knowledge has increasingly become important in the development of prevention and health promotion interventions and legitimising the decisions on which interventions are considered relevant for public health policy [13, 26]. And in the last decades, the scientific knowledge used has also become more and more sophisticated (cf. [24]). With the growing knowledge about diseases and chronic illnesses, more became known about health determinants, health behaviours, risk factors and their distribution among populations. As the public health field uses and produces this kind of knowledge, it has contributed to this increasing sophistication. With its features being among others the collection and use of epidemiological data, population surveillance and other forms of empirical quantitative assessment [4], there is an increasing understanding of complex health determinants and emphasis on establishing the effectiveness of a broad range of interventions (see e.g., [4, 5, 7, 28]).

Tools that are often used for these purposes are screening methods and risk profile assessments. The screening methods and risk profile assessments function as 'technologies of trust' (cf. [26]), as the numerical outcomes they produce have become associated with objectivity and legitimisation $[1,26]$. However, these tools or instruments are more than just measuring tools; they are components in the interventions like behavioural counselling or health education. In other words, screening methods and risk profile assessment are not neutral. Whereas these instruments create effects, they also reduce the target population and thereby decrease the effects. As they are used to screen and select risk groups for prevention and health promotion programmes, they legitimise medical intervention or treatment to be undertaken (cf. [14]) as they objectify the health status of individuals and remove it from all subjective interpretation and personal discretion. But at the same time, these same instruments decrease the effects. As these instruments are being used to screen and select individuals, eligible for intervention, they exclude individuals that do not match the criteria and reduce the target population thereby decreasing possible effects.

Sociologists have critiqued the emphasis on objectivity in medicine in general and in public health in particular. According to their arguments, the use of 
epidemiological knowledge and tools such as screening methods and risk profile assessments in medicine and public health define 'health' a matter of individual responsibility and control and therefore stigmatises and victimises selected individuals for having a disadvantaged or 'deviant' health status [3, 12, 18-20]. In other words, with health to be considered a matter of one's self-control, individuals are summoned to live healthy lives, as it is the morally right thing to do. In fact, it is implied that one is to blame for one's future illnesses provided that one uses the epidemiological knowledge that is provided and one alters one's lifestyles accordingly. As Lupton has argued, such an approach to health assumes that people are 'passive patients' that need to be made aware-or better educated-of the prospective threats that are associated with their lifestyle choices and need to be assisted in altering their lifestyles [18-20]. In addition, Horstman and Houtepen [11] have argued that this view, which has been dominant in critiques about public health, is itself criticised as it does not do justice to individual preferences and choice. In fact, it suggests that people do not have agency and are victimised and stigmatised by prevention and health promotion programmes and their instruments, whereas health care professionals and participants in fact do have agency and voice.

In this paper, in addition to Lupton and others we focus on the functioning of screenings instruments and risk profile assessments and the role they fulfil in prevention and health promotion programmes, with that difference that we focus on the active role patients fulfil in the interpretation of such instruments and the outcomes and labels they produce. Questions we explore in this paper are: How do these instruments construct a target population for prevention and health promotion programmes as well as construct the health risks that should be targeted? How is the actual intervention shaped by the individuals that are detected by means of these instruments? How do these individuals interpret such instruments, their outcomes and labels? Answers to these questions might relativise Lupton's argument, that patients are victimised by the instruments used in prevention and health promotion interventions. The meaning and definitions patients give to instruments and the outcomes and labels they produce, have to be taken into account in order to come to understand the concept of effectiveness of prevention and health promotion interventions.

The emphasis on establishing effectiveness in prevention and health promotion is the result of its rationalised character (cf. [14, 23]), which underscores a univocal significance of 'effectiveness' of interventions. In a rationalistic approach, the effectiveness-or better the success of interventions-is reflected by the impact these interventions have on improving the health status of their participants. In successful interventions the well-aimed stimuli of health educational advice, are considered to result in behavioural change in participants in a linear way leading to the improvement of their health status. Alternative interpretations of what 'good health' or 'wellbeing' is according to participants are considered less or not important and subsequently become invisible, as these do not fit the parameters of the screening methods and risk profile assessments used in establishing the effectiveness of interventions. However, numerous interventions have shown no or minimal effects [17, 21, 22, 33]. And often this lack of effectiveness is classified as a problem of performance (see e.g., [2, 10, 27] in [16]). 
Whereas Lupton and others have placed themselves in opposition to medicine in general and public health in particular, in this paper we do not look at prevention and health promotion from a normative point of view nor do we look at it as a 'problem of performance'. We try to overcome these aforementioned approaches and engage with prevention and health promotion by taking the internal dynamics and logics of medicine in practice seriously (cf. [31]). In order to take both the 'problems' of effectiveness and performance seriously the screening methods and risk profile assessments need to be followed.

\section{Research Methods}

\section{The Quattro Study ${ }^{1}$}

The data presented here comes from an ethnographic study that paralleled the Quattro Study, which was a pragmatic randomised controlled trial on the effectiveness of multidisciplinary patient care teams in primary care for the secondary prevention of cardiovascular diseases (CVD) administered in primary health care centres in deprived neighbourhoods or Rotterdam and The Hague, the Netherlands. The intervention consisted of the formation of a Quattro-care team in general practice composed of a general practitioner (GP), a GP assistant, a practice nurse and a peer health educator for the provision of intensified preventive care to high-risk patients. This intensified preventive care consisted of patient-tailored health education about the risk factors associated with CVD, such as hypertension, diabetes mellitus, hypercholesterolemia, smoking and obesity provided by the practice nurse and/or peer health educator. Structural CVD risk profile assessments, containing physical examinations of weight, height and blood pressure and blood tests on cholesterol and glucose levels, were used to assess the intervention participants' risk profiles. And multidisciplinary team meetings, to be organised by the Quattro-care team professionals, functioned as a manner for jointly establishing treatment plans for intervention participants and monitor the reduction in their risk profiles based on the particular knowledge and abilities of each professional.

In order to evaluate the effectiveness of multidisciplinary patient care teams in primary care, the intervention followed the procedures of a pragmatic randomised controlled trial (RCT), which aimed to measure the effectiveness of the intervention under natural-non-experimental-conditions. For this programme, the trial researchers selected patients from the centres' electronic patient records with a modifiable part of the absolute 10-year risk on cardiovascular diseases (CVD) of at least 20\%. People eligible for the Quattro Study (1) had to live in deprived neighbourhoods, as defined by the Dutch National Association of General Practitioners (LHV); (2) were to be aged between 18 and 70 years of age; (3) have a medical history of one or more risk factors for cardiovascular diseases (CVD) (e.g., smoking; obesity); (4) a first degree relative with a history of CVD

\footnotetext{
${ }^{1}$ For a more elaborate description of the intervention, study methods including its methodological justification and overviews of its results see: El Fakiri [8].
} 
before his/her 60th birthday; hypertension; hypercholesterolemia; Diabetes Mellitus type II; myocardial infarction; angina pectoris; peripheral arterial disease, heart failure, CVA or TIA.; and (5) having an absolute 10-year risk for CVD, higher than $20 \%$ after blood tests and physical examinations at baseline (document selection criteria addendum 2).

Eligible patients were randomly assigned to three groups. Patients in the intervention group obtained Quattro-care and three monthly assessments of their risk profile for CVD. Patients from the first control group (A) received usual GP care and three monthly risk assessments and the GP as well as the patient were informed about the results of these measurements. It was thought to be ethically and practically unacceptable to assess a risk profile and not to inform the patient and GP about the results. However, this approach of assessing risk and informing patients and GP was thought to interfere with daily practice and bias the results. Therefore, a second blinded control group (B) was needed to quantify the effect of the risk assessments. This group received usual GP care and was measured once at the end of the study. The research team assessed the effectiveness of the multidisciplinary collaboration with the returned patient monitoring data by comparing patients from the intervention group with those from the first control group after 1-year follow-up with regard to the reduction achieved in the 10-year absolute risk of developing CVD. The second control group, aimed to quantify the effect of structured risk profile assessments performed in the first control group, was compared with the first control group.

\section{The Ethnographic Study}

In order to analyse how this prevention programme was shaped in practice, we observed how the practice nurses provided the patient-tailored health education and performed the structural CVD risk profile assessments and how these facilitated the patients in achieving lifestyle modifications. By means of participant observations $[6,29]$, we observed four out of seven practice nurses assigned to the project in their daily work, each for five workdays each, from April 2003 till December 2004. One practice nurse refused cooperation; one practice nurse was on maternity leave and one was not yet in function. During the observations no notes were made, because note taking was felt to intrude on the interactions between the practice nurses and their patients and colleagues. During these observations a total of 63 patient consultations were attended. Because of the recurrent nature of the programme's follow-up, the observations also contain succeeding follow-up consultations of patients. Fieldnotes were made immediately after leaving the health care centres. Throughout each observation it was possible to ask questions or to request clarification. In addition, a total of five interviews were held with the practice nurses: two non-audio-taped semi-structured face-to-face interviews due to objections to audio-taping and three audio-taped semi-structured face-to-face interviews. The interviews were held at the health care centres and lasted approximately one and a halve hour. In all interviews, the practice nurses were asked about their experiences with the Quattro programme, their dealings with patients in the programme and their activities in the primary health care centres. All interviews were transcribed immediately after the interviews. 
The patients in the observed consultations were invited to take part in the Quattro programme, as they were all at high risk for developing CVD and not yet properly managed for hypertension, hypercholesterolemia and/or diabetes mellitus type II. All patients were registered at the health care centres and were living in the deprived neighbourhoods the health care centres provided their services to. During the patients consultations observations were made about how both the practice nurses and patients participated in this health promotion programme. Whenever possible, informal conversations with the patients took place about the possibility and difficulties incorporating the recommended lifestyle adjustments into their lives. With three patients audio-taped semi-structured face-to-face interviews were held to go more in-depth into their motives for participating in prevention programmes and the difficulties participation may portray. The interviews lasted approximately one hour and were held at the patients' homes. The interviews were transcribed immediately after the interviews.

In this study the process of analysis took place in a cyclic manner. Emerging insights and themes were jotted down, and were taken into account during succeeding observations, interviews and final analysis. During the final analysis, all transcripts, written records, and personal jot notes were analysed more in-depth. We analysed all information thematically, establishing overarching categories through identifying and coding all pieces of information. By means of developing overarching categories (taxonomy) overall descriptions of the information could be made in more general terms.

\section{Results}

The Role of Inclusion Criteria in the Selection and Enrolment of Eligible Individuals

We start our analysis of the role of screening methods and risk profile assessments by looking at the selection of people eligible for prevention interventions. Screening methods and risk profile assessments are not neutral. What we saw in our ethnographic study was interaction between the inclusion criteria as example of such instruments and selected individuals. The inclusion criteria did not determine which individuals participated and which did not. The selected individuals were seen to have an active role in the selection and enrolment of the intervention and therewith they related to the inclusion criteria. The inclusion criteria were part of the individuals' legitimisation to enrol or to reject their participation. Enrolment or rejection of participation thus was more than just the projection of the individuals' opinion on the outcomes of the inclusion criteria; enrolment or rejection of participation was the resultant of the individuals' opinion that was formed in interaction with the inclusion criteria.

For the selection of people eligible for the Quattro intervention, the inclusion criteria were important as they explicated the risk to CVD people had and indicated the danger of having a CVD event in future. The inclusion criteria enrolled only those individuals who were identified with the risk to CVD. What we saw was that 
people actively negotiated the identity of patienthood the inclusion criteria ascribed them.

'what does a $15 \%$ risk for CVD matter when I am feeling well'. [...] the patients with diabetes [...] they clearly have diabetes; a high sugar level, that is more concrete to deal with (interview practice nurse 27-01-2006)

In fact, the inclusion criteria legitimatised the decision to enrol into the prevention programmes or not. The inclusion criteria thus created effects, as people enrolled into the intervention, and at the same time they reduced the group of eligible people thereby decreasing the ultimate effect. In the Quattro programme people enrolled as "they thought that their GP had registered them for the study; that they were screened by their GP. They found it was important to take part, as they thought they had a high risk" (interview practice nurse 27-01-2006). The fact that these people had become selected for the Quattro programme suggested that their enrolment was important as they were 'at risk'. The individuals who enrolled, identified themselves with the danger to their health these inclusion criteria portrayed, as well as identified themselves with the classification of patienthood the inclusion criteria ascribed them.

On the other hand, the inclusion criteria also obstructed individuals to take part in the intervention. In fact, the individuals who felt they were defined as people they felt they were not declined their enrolment. For example, one of the people selected to take part in the trial refused to participate in the trial, because he refused being defined as ill. The man, in his mid thirties and hardly home from work at four o'clock p.m., explained, while sitting on his couch smoking his hand-rolled tobacco, he had taken blood pressure lowering medication in a very stressful period of his life in which he had to deal with losing his job that caused the rise of his blood pressure levels. "But now I don't take these pills anymore, because that stressful period is over. So, I don't think it is necessary for me to take part" (observation patient selection fase May 2003). Others refused to participate in the programme, as a nurse explained, "because they fiercely objected to the fact they were assumed to live in deprived neighbourhoods. They felt they were defined as people they felt they were not" (observation data manager 28-02-2006).

The south of Rotterdam indeed has some bad neighbourhoods, but would you define this neighbourhood in which the houses sell for up to 400.000 euros a deprived neighbourhood? That's why I don't agree seeing the south of Rotterdam as a deprived neighbourhood (interview patient 14-03-2006)

The enrolment of eligible individuals into interventions can thus be understood as the result of individuals actively accepting or rejecting the definition of patienthood and/or as living in deprived neighbourhoods that these inclusion criteria ascribe to them. The use of inclusion criteria as a method for selecting and enrolling individuals in prevention programmes legitimises the decision to enrol into prevention programmes. On the one hand, the inclusion criteria create effects, as they objectify the individual risk to health individuals have by explicating the conditions that make up that risk, like their genetic make-up and/or physical condition and the neighbourhoods with its related lifestyles people live in. Yet, the 
inclusion criteria reduce the group of eligible people and thus decrease the possible effect of the intervention. The inclusion criteria do not select and enrol all individual who are considered eligible based upon the health risks they have, they only select those individuals 'at risk' that identify with the construction made and are open to adapting their lifestyles to the requirements of the prevention programmes. People thus demonstrate their agency and voice, as they actively decide whether to enrol into prevention programmes or not. And the inclusion criteria provide them the legitimisation for their decision.

\section{The Role of Structural Risk Profile Assessments in the Course of Prevention Programmes}

In the course of prevention programmes, the same movement of creating effects and at the same time decreasing possible effects can be seen for structural risk profile assessments. The structural risk profile assessment did not determine how the patients participated and adapted their lifestyle behaviours or not. Also during the course of the prevention intervention patients were seen to have an active role and therewith they related to the structural risk profile assessments. In fact, the structural risk profile assessments were part of the legitimisation to adapt treatment to the personal circumstances of patients or for patients to adopt the recommended lifestyle behaviours or not. Actual treatment and behavioural change was the resultant of the opinion that were formed in interaction with the inclusion criteria.

In the Quattro Study, the three-monthly structural CVD risk profile assessments (re)established the anticipated chance of having a CVD event in future. Through measuring a patient's weight, length and blood pressure and issuing blood tests on cholesterol and glucose levels, the risk for CVD is defined as the conglomerate of various characteristics of the individual's biology and lifestyle that are to be considered traits or advantages to health. As a practice nursed explained:

Risk factors become more and more important. [...] When a patient has a blood pressure over $160 \mathrm{mmHg}$, you not only look at the height of his blood pressure, you also have to look at other risk factors, like e.g. age, weight, length, smoking and cholesterol. [...] Registering is here of utmost importance. First you have to gather all the data before you can calculate the absolute risk profile of patients (observation researcher 09-03-2004)

The CVD risk profile also defines which parts of the risk for CVD are changeable through lifestyle modifications and which are not. For example: "a patient's age and gender [...] are not modifiable; they are fixed variables in the risk for CVD" (interview researcher 26-01-2006). Whereas the individual's biology is genetically determined, the other variables in the risk for CVD—a person's weight, blood pressure, cholesterol and glucose levels - are modifiable through diet, physical exercise and smoking cessation. Lifestyle, however, does not let itself modify that easy as it involves the continuous abandonment of habits and lifestyle patterns that have been there for long. In other words, with the structural risk profile assessments explicating the unique constellation of a patient's risk for CVD, these assessments drive an individualised management of risk. The personal problems participants 
have to deal with on a daily basis determine the extent of the improvements in their health. As a practice nurse illuminated:

The practice nurse is the person for that inventory. [...] A GP doesn't see the real problems patients deal with; he only sees e.g. a high blood pressure and not the patients' daily personal problems. To be able to supervise these patients, a practice nurse needs to understand these problems patients deal with (observation intervention progress meeting 20-04-2004)

The structural assessment of participants' risk profiles created effects as they visualised the risk behaviours that made up participants' risk profiles. And at the same time, as the structural risk profile assessments measured the effect of the individualised management of risk on the reduction of the CVD risk in participants, they reduced the possible impact of the intervention as they allowed for the adaptation of the treatment plans depending upon the outcomes of the measurements. Although this was not intended in the original design of the intervention, the risk profile assessments allowed the practice nurses to create a hierarchy in risk factors in individual participants, as they believed not all risk factors could be tackled all at once. In fact, the risk profile assessments made it possible to change those risk behaviours that made up participants' risk profile in which reductions in CVD risk could be achieved with minimal efforts. In other words, the practice nurses negotiated the original purpose of the risk profile assessments as this was not workable in daily practice.

What we saw in participants was that, as these assessments visualised the progress participants were able to make in reducing their CVD risk and improving their health status, they functioned as a motivator for achieving the recommended lifestyle changes. In other words, these assessments created effects as they monitored the effects of the patients' lifestyle choices. For example:

The practice nurse puts on the cuff and starts taking the patient's blood pressure. Both the patient and the practice nurse look at the sphygmomanometer. When she is finished, she takes off the cuff and writes the results on a piece of paper. 'Is it alright now?' the man asks. The practice nurse nods her head and says it looks fine (observation patient consultation 04-11-2004)

And as a patient tells one of the practice nurses about loosing weight:

'Indeed, you have lost almost three kilos in two weeks time. How do you keep up?' The female patient tells she does follow a diet since two weeks now [...] 'Good, and your kids?' 'They get their own food'. The female patient twinkles when she is telling the practice nurse. 'It feels good. My clothes do fit much better already.' And she lifts up her dress by the shoulder seams [...] (observation patient consultation 14-07-2004)

Yet, we also saw that these structural risk profile assessments reduced the groups of patients adopting new lifestyle behaviours and thereby decreasing the ultimate effect of the intervention. In fact, the patients negotiated the lifestyle modifications that the structural risk profile assessments envisaged in order to reduce the risk of cardiovascular diseases. As the structural risk profile assessments visualised their 
CVD risk profile as a conglomerate of risk factors, these assessments enabled them to make choices in the behavioural changes they were recommended to make and in what order. For example as illustrated in a fragment: "I regularly walk the dog now and take my medication when I'm supposed to, but I still eat my usual mash. I don't like the extra fuss. I told the practice nurse from the beginning I'm not changing that!" (conversation patient 14-07-2004). So, the risk profile assessments as they objectify the risk to CVD have effects and construct the behaviour of patients. These assessments thus prove to be a constructive force. On the one hand, they motivate patients to continuously achieve improvements in their health. On the other hand, they decrease the ultimate effect of prevention interventions as these assessments give patients agency and voice, as they are facilitated to actively decide upon the lifestyle changes they want to incorporate into their lives.

\section{Discussion}

In this paper, we analysed the role screening methods and risk profile assessments fulfil as part of a prevention and health promotion programme in the selection, enrolment and participation of participants. Our analysis showed, that screening methods and risk profile assessments create effects as they objectify health risks and/or the health status of individuals, i.e., they select the individuals 'at risk' and indicate the lifestyle modifications these people are required to make in order to improve their health. Yet, these instruments also reduce the group of participants thereby decreasing the possible effect of interventions, as they provide the legitimisation for people to make choices to whether they enrol or not and what lifestyle changes they incorporate into their lives. The outcomes of the risk profile assessments function as a tool for participants for making these choices. The assessment of participants' risk profiles present the risk to health as a collection of smaller parts, from which participants are enabled to choose the changes to their lifestyles. In other words, these instruments present a space of interaction, in which agency is distributed across the practice nurses, the participants and the risk profile assessments. Decisions were not just made upon the projection of the outcomes of these instruments; decisions that were made by both the patients and practice nurses were the resultant of their opinions on these outcomes that were formed in interaction with the instruments.

Our results have two important implications. Firstly, prevention and health promotion programmes have a more limited outreach than is assumed when interventions are developed. Such programmes only exhort a selective group of their target populations to participate. In addition to Howson [12], who argued that screening methods and risk profile assessment are instruments of inclusion and exclusion, screening methods and risk profile assessments only include those people that identify with the construction of patienthood that is made. Those people who do not identify with the construction are excluded but may very well benefit from health promotional activities too.

Secondly, the effects of screening methods and risk profile assessments generated in prevention and health promotion programmes should be considered as effects of 
such interventions. The use of scientific knowledge and method feeds the notion that improvement in health can be rationalised and numerically established and is the only objective proof of the interventions' effectiveness, as the effectiveness is evaluated through comparing the new interventions and/or treatments versus regular care. Subsequently, this leaves no room for other effects-i.e., the positive effects of risk profile assessments on health improvement in prevention interventions $[8,9]-$ that are found in interventions, as they simply do not count as genuine effects. These effects are considered merely part of regular care of which the effectiveness is not evaluated. Result of such a narrow focus on effectiveness is that the aforementioned effects thus remain invisible, as they are considered outside of the scope of the evaluations.

We therefore argue, in concordance with Horstman and Houtepen [11], that in order to establish the effectiveness of interventions pragmatic forms of evaluation should be applied. Pragmatic evaluations provide the opportunity to broaden up the scope of effectiveness in evaluations in which all effects of interventions can be incorporated and can be classified as effects of the interventions. Pragmatic evaluations allow for the incorporating of multiple evaluation methods [25, 32] that allow for the incorporation of the ongoing dynamics in interventions when performed in practice, as interventions will always be localised and contextualised in practice [16, 32]. The way in which screening methods and risk profile assessments construct behaviour thus can than also be evaluated as genuine effects of prevention and health promotion interventions.

In conclusion, it is our contention that there should be more attention and appreciation for the role screening methods and risk profile assessments fulfil in generating health effects. Therefore, a broader notion of effectiveness should be obtained in prevention and health promotion. Moreover, screening methods and risk profile assessments fulfil an important role in the empowerment of patients, as these instruments legitimise decisions on enrolment, participation and behavioural change and facilitate them to make autonomous decisions about their care.

Acknowledgments We would like to thank the health care professionals and patients, who participated in the Quattro Study, deeply for the time and effort they put in having the first author as a participant observer. In addition, we would like to express great gratitude to the Quattro Study researchers for the opportunity of conducting this ethnographic study alongside their research project and their openness and feedback during the data collection period. We would also like to thank Roland Bal and Marleen Foets for their input and feedback when writing this paper.

Open Access This article is distributed under the terms of the Creative Commons Attribution Noncommercial License which permits any noncommercial use, distribution, and reproduction in any medium, provided the original author(s) and source are credited.

\section{References}

1. Bartholomée, Y. (2004). Society as a Laboratory; Donald T. Campbell and the history of social experimentation. Psychologische, Pedagogische en Sociologische Wetenschappen. Groningen: Rijksuniversiteit Groningen. 
2. Blasinsky, M., Goldman, H. H., et al. (2006). Project IMPACT: A report on barriers and facilitators to sustainability. Administration and Policy in Mental Health and Mental Health Services Research, 33(6), 718-729.

3. Bodenheimer, T., Lorig, K., et al. (2002). Patient self-management of chronic disease in primary care. Journal of American Medical Association, 288(19), 2469-2475.

4. Childress, J. E., Faden, R. R., et al. (2002). Public health ethics: Mapping the terrain. Journal of Law, Medicine \& Ethics, 30, 170-178.

5. Coughlin, S. S. (2006). Ethical issues in epidemiologic research and public health practice. Emerging Themes in Epidemiology, 3, 16. doi:10.1186/1742-7622-3-16.

6. Creswell, J. W. (2003). Research design; qualitative, quantitative, and mixed methods approaches. Thousand Oaks: SAGE Publications, Inc.

7. Edwards, N., Mill, J., et al. (2004). Multiple intervention research programs in community health. Canadian Journal of Nursing Research, 36(1), 40-54.

8. El Fakiri, F. (2008). Prevention of cardiovascular diseases in deprived neighbourhoods. Rotterdam: Institute Health Policy and Management, Erasmus MC.

9. El Fakiri, F., Hoes, A. W., et al. (2008). Process evaluation of an intensified preventive intervention to reduce cardiovascular risk in a multiethnic patient population. European Journal of Cardiovascular Nursing, 7(4), 296-302.

10. Getrich, C., Heying, S., et al. (2007). An ethnography of clinic "noise" in a community-based, promotora-centered mental health intervention. Social Science and Medicine, 65(2), 319-330.

11. Horstman, K., \& Houtepen, R. (2005). Worstelen met gezond leven: ethiek in de preventie van harten vaatziekten. Het Spinhuis: Amsterdam.

12. Howson, A. (1998). Surveillance, knowledge and risk: The embodied experience of cervical screening. Health, 2(2), 195-215.

13. Hunter, D. J. (2003). Public health policy. Cambridge: Polity Press.

14. Jansen, Y. J. F. M. (2010). The taming of chance and the actual practice of prevention; rationalised prevention and 'the social'. In T. Mathar \& Y. J. F. M. Jansen (Eds.), Health promotion and prevention programmes is practice; how patients' health practices are rationalised, reconceptualised and reorganised (pp. 147-169). Bielefeld: Transcript Verlag.

15. Jansen, Y. J. F. M., de Bont, A., et al. (2007). Tailoring intervention procedures to routine primary health care practice; an ethnographic process evaluation. BioMed Central Health Services Research, 7, 125. doi:10.1186/1472-6963-7-125.

16. Jansen, Y. J. F. M., \& Foets, M. M. E., et al. (2009). The contribution of qualitative research to the development of tailor-made community-based interventions in primary care: A review. European Journal of Public Health, 1-7 [Epub ahead of print]. doi:10.1093/eurpub/ckp085.

17. Kooiker, S., \& van der Velden, K. (2007). Een nuchtere kijk op gezond gedrag; Vier thema's voor gezondheidsbevordering. Sociaal en Cultureel Planbureau: Den Haag.

18. Lupton, D. (1993). The construction of patienthood in medical advertising. International Journal of Health Services, 23(4), 805-819.

19. Lupton, D. (1993). Risk as moral danger: The social and political functions of risk discourse in public health. International Journal of Health Services, 23(3), 425-435.

20. Lupton, D. (1997). Consumerism, reflexivity and the medical encounter. Social Science and Medicine, 45(3), 373-381.

21. Mackenbach, J. P., \& Stronks, K. (2002). A strategy for tackling health inequalities in the Netherlands. British Medical Journal, 325(7371), 1029-1032.

22. Mackenbach, J. P., \& Stronks, K. (2004). The development of a strategy for tackling health inequalities in the Netherlands. International Journal for Equity in Health, 3, 11. doi: 10.1186/1475-9276-3-11.

23. Mathar, T. \& Jansen, Y. J. F. M. (Eds.) (2010). Health promotion and prevention programmes is practice; how patients' health practices are rationalised, reconceptualised and reorganised. VerKörperungen/MatteRealities-Perspektiven empirischer Wissenschaftsforschung. Bielefeld, Transcript Verlag.

24. May, C. (2009). Mundane medicine, therapeutic relationships, and the clinical encounter: Current and future agendas for sociology. In B. Pescosolido, J. K. Martin, J. D. McLeod, \& A. Roggers (Eds.), Handbook of the sociology of health, illness, \& healing: A blueprint for the 21st century. New York: Springer.

25. Nutbeam, D. (1998). Evaluating health promotion-Progress, problems and solutions. Health Promotion International, 13(1), 27-44. 
26. Porter, T. M. (1995). Trust in numbers; the pursuit of objectivity in science and public life. Princeton: Princeton University Press.

27. Rousseau, N., McColl, E., et al. (2003). Practice based, longitudinal, qualitative interview study of computerised evidence based guidelines in primary care. British Medical Journal, 326(7384), 314.

28. Shim, J. K. (2005). Constructing 'Race' across the science-lay divide: Racial formation in the epidemiology and experience of cardiovascular disease. Social Studies of Science, 35(3), 405-436.

29. Spratley, J. P. (1980). Participant observation. Fort Worth: Harcourt Brace College Publishers.

30. Timmermans, S., \& Berg, M. (2003). The gold standard; the challenge of evidence-based medicine and standardization in health care. Philadelphia: Temple University Press.

31. Timmermans, S., \& Haas, S. (2008). Towards a sociology of disease. Sociology of Health \& Illness, $30(5), 659-676$.

32. Tones, K. (2000). Evaluating health promotion: A tale of three errors. Patient Education and Counseling, 39(2-3), 227-236.

33. Uitewaal, P. J. M. (2003). Type 2 diabetes mellitus and Turkish immigrants: An educational experiment in general practice. Rotterdam: Institute Health Policy and Management, Erasmus MC. 